\title{
Web 2.0 et musées : les nouveaux visages du visiteur
}

\section{Proposition pour la rubrique « Digital »}

\section{Rémi MENCARELLI}

Maître de Conférences - HDR IAE de Dijon - Université de Bourgogne LEG (CERMAB)

\section{Adresse professionnelle :}

IAE de Dijon - LEG/CERMAB

Université de Bourgogne Pôle d'Economie et de Gestion 2 boulevard Gabriel BP 26611

21066 Dijon cedex

Tel : 0380393506

Fax : 0380395443

E-Mail : remimencarelli@yahoo.fr

Adresse personnelle :

$$
\begin{aligned}
& \text { 4, rue Gagnereaux } \\
& 21000 \text { Dijon }
\end{aligned}
$$

Tel : 0380715522

Port : 0628070733

E-Mail : remimencarelli@yahoo.fr

\author{
Mathilde PULH \\ Maître de Conférences \\ IUT de Dijon - Université de \\ Bourgogne \\ LEG (CERMAB)
}

Adresse professionnelle :

$$
\begin{gathered}
\text { IUT de Dijon } \\
\text { LEG/CERMAB } \\
\text { Université de Bourgogne } \\
\text { Boulevard Dr. Petitjean } \\
\text { BP 17867 } \\
\text { 21078 Dijon Cedex } \\
\text { Tel : } 0380396470 \\
\text { Fax : } 0380396479
\end{gathered}
$$

E-Mail : mathilde.pulh@u-bourgogne.fr

Adresse personnelle :

$$
\begin{gathered}
12 \text { avenue de Spy } \\
21220 \text { Gevrey Chambertin } \\
\text { Tel : } 0380652355 \\
\text { Port : } 0683182405
\end{gathered}
$$

E-Mail : mathilde.pulh@,orange.fr 


\section{Web 2.0 et musées : les nouveaux visages du visiteur}

Résumé : L'intégration croissante des techniques issues du Web 2.0 par les musées illustre les profondes modifications que ces structures ont engagées dans la construction de leur relation avec le public. Le recours à ces dispositifs, basés sur une logique de participation, conduit à impliquer le visiteur, traditionnellement tenu à distance, dans toutes les étapes de la mise en œuvre de l'offre muséale sur Internet. Grâce à un examen attentif des sites et des outils proposés par les structures muséales, différents rôles désormais dévolus au public ont pu être identifiés. L'émergence de ces nouvelles figures du visiteur vient interroger à plus long terme l'identité et la légitimité des musées eux-mêmes.

Mots clés : musées, Web 2.0, marketing participatif, visiteur acteur, légitimité

\section{Web 2.0 and museums: the new faces of the visitor}

Summary: The increasing integration of techniques from Web 2.0 by the museums illustrates the profound changes that these structures have realized in building their relationship with the audience. The use of these devices, based on a logic of participation, leads to involve the visitor, traditionally rather kept at a distance, on all stages of the implementation of the museum offer on the Internet. Through a careful examination of sites and features offered by the museal structures, different roles now devolved to the public have been identified. The emergence of these new "faces" of the visitor comes to question, in the longer-term, about the identity and the legitimacy of the museums themselves.

Keywords: museums, Web 2.0, participatory marketing, visitor actor, legitimacy 


\section{Introduction}

Depuis une vingtaine d'années, la sphère muséale ne cesse d'alimenter les débats, notamment quant à la définition de ses responsabilités, de ses contenus, de ses outils de médiation ou encore de ses cibles. En effet, si les missions de conservation et de préservation des œuvres ont longtemps dominé les activités des musées, la mission de diffusion des collections vers le grand public apparaît désormais déterminante.

Dès les années quatre-vingt, plongées dans un contexte de concurrence exacerbée et de forte contrainte budgétaire, les structures muséales ont pris conscience de la nécessité d'attirer et de fidéliser un plus grand nombre de visiteurs. Cependant, ce public, même ardemment convoité, a longtemps été cantonné à une exigence purement contemplative et à une valorisation principalement esthétique ou intellectuelle de son expérience de consommation muséale.

La réalité semble aujourd'hui toute autre. Certaines organisations reconnaissent en effet la diversité de leurs publics et multiplient les initiatives pour leur apporter des sources de valorisations nouvelles. Elles n'hésitent pas à redéfinir et enrichir l'expérience proposée. A ce titre, l'intégration des nouvelles technologies au sein des projets muséaux a marqué un tournant, en permettant une nouvelle forme de relation entre le musée et ses visiteurs. Les premières expérimentations (à l'instar de la Cité des Sciences et de l'Industrie dans les années quatre-vingt-dix) ont reposé sur le développement de formes de multimédia interactif au cœur des expositions elles-mêmes (audio guide, bornes interactives, vitrines parlantes...) établissant ainsi une forme d'échange inédit entre le musée et ses visiteurs (11).

Actuellement, loin de se démentir, cette tendance est en train de s'accélérer. En effet, au-delà d'une interaction in situ, les institutions cherchent à développer une forme d'interactivité nouvelle à travers la mobilisation des techniques relatives au Web 2.0 ${ }^{1}$. Elles n'hésitent plus à donner à leurs visiteurs davantage d'influence, voire de maîtrise, sur la définition de l'expérience proposée via Internet. Cette évolution des pratiques est loin d'être anecdotique car elle semble amener à une redéfinition profonde de la relation qui peut se tisser entre une offre culturelle et son public avec l'émergence d'un visiteur désormais acteur de l'offre muséale. Elle illustre en parallèle la montée en puissance des «pro-am » (professionnelsamateurs) de plus en plus sollicités par les organisations pour leurs capacités à diffuser de l'information, à produire de la connaissance voire à générer des œuvres (6). Cette tendance inscrit pleinement les musées dans une logique de marketing collaboratif, fondé sur un

\footnotetext{
${ }^{1}$ Le web 2.0 peut se comprendre comme une « architecture de la participation » où les utilisateurs ne sont plus de simples récepteurs passifs mais peuvent prendre une part active à de la production de contenus (5).
} 
processus de co-construction de valeur entre l'entreprise et le consommateur (2). Cependant, la mise en œuvre d'une telle logique s'accompagne également d'une réinterprétation des rôles à la fois du public et des musées.

L'objectif de cette réflexion est ainsi d'interroger les rôles actuellement dévolus au visiteur à travers l'utilisation des outils 2.0 déployés par les musées. Grâce à un examen attentif des sites et des dispositifs proposés par ces organisations, nous souhaitons montrer comment le visiteur peut à présent être impliqué dans toutes les étapes de la mise en œuvre de l'offre muséale sur Internet : de la médiatisation du contenu, en passant par la définition ou la redéfinition des outils de médiation, en allant jusqu'à une action possible en termes de création de l'œuvre culturelle elle-même. Nous illustrerons chacun de ces rôles, du plus périphérique au plus central, à partir d'exemples représentatifs. Enfin, en conclusion, après avoir identifié les objectifs poursuivis par les responsables muséaux, nous questionnerons l'impact de ces pratiques sur la nature de la relation entre le visiteur et le musée et, plus globalement, sur l'identité, le rôle et la légitimité de ce dernier.

\section{Le visiteur, nouveau « responsable de la communication » des structures muséales?}

Les musées ont investi depuis déjà de nombreuses années l'univers Internet, ayant parié très rapidement sur la capacité de diffusion du Web pour capter de nouveaux publics ou entretenir une relation privilégiée avec leurs cibles les plus fidèles. L'intégration plus spécifique des techniques 2.0 dans cette offre virtuelle a ainsi consisté, dans un premier temps, à sortir d'un schéma de communication unilatérale (entièrement définie et contrôlée par le musée) pour pouvoir profiter du potentiel de chaque internaute. Les premiers pas du marketing participatif muséal sur Internet ont donc concerné cet élément, certes périphérique (par rapport à la définition du contenu proprement dit), mais non négligeable pour favoriser les connexions et les échanges au nom du musée.

Certaines institutions, à l'instar du MoMA de New York, ont donc compris l'intérêt d'assurer une présence proactive sur le web et, plus précisément sur des sites adossés à des réseaux sociaux, afin de mobiliser leurs publics comme autant de vecteurs de communication :

- L'institution américaine a ainsi utilisé You Tube pour créer le MoMAVideos Channel, site sur lequel sont disponibles plus de 250 vidéos, dont certaines ont fait l'objet de centaines de milliers de visionnages, comme le «Tim Burton MoMA spot» regardé plus de 250000 fois (http://www.youtube.com/user/MoMAvideos). 
- Le musée new-yorkais a également investi Facebook depuis mars 2008 et compte près de 886000 «amis», avec une communauté présente et active (http://www.facebook.com/home.php\#!/MuseumofModernArt). Les mots «musée » ou «museum» se révèlent d'ailleurs être des clés d'entrée à des milliers de compte Facebook d'institutions muséales, des plus modestes aux plus renommées, qui proposent de partager, outre des informations traditionnelles, des photos, des articles, des vidéos et des événements.

Ces outils, désormais classiques du Web 2.0, permettent aux musées de se familiariser avec le marketing participatif, tout en gardant un contrôle important sur les contenus générés.

A côté de ces dispositifs, certaines initiatives jouent davantage sur l'implication du public dans la diffusion, voire la définition des messages à transmettre. Les musées ne se contentent plus de miser sur de simples plateformes de relais d'information, mais cherchent à réellement établir une stratégie d'approbation ou de facilitation des actions de communication pour s'ancrer au final dans une logique de co-promotion (1). Dans cette perspective, les modalités d'action sont diverses et variées de la part des structures muséales.

Devant la forte popularité des blogs, plusieurs organisations ont adopté cet outil de diffusion au sein de leur site. C'est notamment le cas du Smithonian American Art Museum avec son blog «Eye Level » qui permet des échanges avec son public (http://eyelevel.si.edu/). Certains musées vont plus loin en invitant leurs visiteurs à laisser des commentaires sur un blog mais en version audio ou vidéo. C'est le cas du Mattress Factory Art Museum de Pittsburg qui a créé l'outil MFIConfess où chaque visiteur peut laisser sa propre dédicace vidéo (http://artyoucangetinto.blogspot.com/2009/02/some-mficonfess-videos-from-this.html).

Dans une perspective similaire, d'autres structures, à l'instar du Brooklin Museum, proposent à leurs visiteurs de déposer leurs commentaires via Twitter et de recevoir des réponses et des réactions (http://twitter.com/brooklynmuseum).

Enfin, pour certains musées, il s'agit de véritablement favoriser une interaction en allant jusqu'à incorporer les messages créés par les visiteurs pour alimenter leurs campagnes de communication. Ainsi, en janvier 2009, le Metropolitan Museum of Art (MET) a-t-il lancé une campagne innovante sous le slogan «It's time we MET !». Cette opération consistait à demander aux visiteurs de déposer sur Flickr leurs photographies symbolisant des moments vécus en famille ou entre amis au sein du musée, puis à sélectionner parmi les milliers de clichés reçus ceux qui allaient être primés et insérés officiellement dans la campagne de communication institutionnelle du musée. 
Ces exemples illustrent parfaitement le fait que les musées engagent plus ou moins directement les visiteurs dans la définition de leurs outils de communication. Si certains musées cherchent uniquement à mobiliser le consommateur comme un agent de transmission d'informations définies au préalable par l'institution, d'autres musées permettent aux visiteurs de compléter d'organiser, voire de produire cette information. Il s'agit alors pour les structures muséales de renforcer l'attachement du public à leur égard afin de pouvoir notamment capitaliser sur un bouche-à-oreille positif. Il convient, cependant, de garder à l'esprit, que derrière une logique participative affichée, les musées contrôlent le plus souvent les contenus, choisissant notamment les éléments à publier ou non afin de gérer leur «eréputation ».

\section{Le visiteur, nouveau « conservateur » des musées en ligne?}

Les dispositifs précédemment évoqués, s’ils sont collaboratifs, se limitent à une intervention du visiteur sur des éléments en marge de l'offre muséale elle-même. Certaines institutions ont, quant à elles, décidé de franchir un pas supplémentaire en permettant l'action et la participation du visiteur de façon plus centrale. Dans le cadre de cette démarche collaborative « intermédiaire », il ne s'agit pas encore pour les musées de laisser les visiteurs définir le contenu de leur offre. Certains outils vont plutôt leur permettre d'endosser un rôle traditionnellement dévolu au conservateur, celui de structurer librement ce contenu et d'en définir notamment les éléments de médiation.

La mise en place d'outils de «folksonomie » en constitue un parfait exemple. Cette démarche, qui combine l'idée de taxinomie (règles de classification) et de peuple (folk), repose sur la création par des non-spécialistes d'un système de classification collaboratif décentralisé et spontané. Elle s'oppose ainsi aux systèmes de classification « experts » qui contraignent les non-spécialistes par des termes prédéfinis (8).

Pour les musées, la mise en place d'une telle démarche consiste à laisser la main au public quant à la désignation et à la classification des œuvres proposées en leur donnant la possibilité d'associer leurs propres mots clés (tags) aux contenus muséaux mis en ligne. Les structures sont de plus en plus nombreuses à tester les avantages de ce tagging social comme nouveau moyen de décrire et d'accéder à leur collection. Comme le Metropolitan Museum of Art, le Minneapolis Institute of Arts ou encore le Rubin Museum of Art, le Sydney Powerhouse Museum invite ses visiteurs à tagger chaque objet de la collection (http://www.powerhousemuseum.com/collection/database/browsekeywords.php) afin de 
proposer, en parallèle à la terminologie des experts, une classification construite par et pour ses usagers.

Une telle action se révèle être un puissant outil d'appropriation du contenu muséal proposé. En effet, en contrepoids d'un thesaurus scientifique défini par le conservateur, souvent complexe pour le grand public, les musées peuvent faciliter, via le tagging social, la rencontre et la compréhension de leur projet culturel ou scientifique par leurs visiteurs. Ils peuvent alors espérer atteindre une de leurs missions capitales, celle d'éducation du public. Une étude sur les comportements de tagging des visiteurs de sites de musées américains a d'ailleurs montré que $86 \%$ des tags fournis ne correspondaient à aucune étiquette référencée par les musées. Ces données illustrent bien la complémentarité de cette démarche, d'autant plus importante que plus de $88 \%$ de ces tags étaient considérés comme utiles à la recherche par les membres du personnel des musées (10).

Par ailleurs, selon Le Deuff (8), la folksonomie favorise une forme de navigation basée sur la sérendipité : le recours à ces tags non experts est ainsi un excellent moyen de faire découvrir de façon inattendue certains contenus muséaux à des internautes, sans qu'ils les aient volontairement recherchés.

Au-delà d'une appropriation et d'une navigation facilitée, cette démarche peut également être considérée comme un puissant outil de crédibilisation des contenus offerts. Dans la mesure où le visiteur peut catégoriser lui-même les objets auxquels il se confronte, il pourra apprécier plus aisément la valeur de l'offre proposée. Cet outil spécifique du Web 2.0 peut ainsi répondre à une problématique récurrente des institutions : être capable d'effacer les barrières symboliques empêchant l'élargissement du public et l'accessibilité à leurs contenus.

A travers ces différentes démarches émerge bel et bien l'idée d'un musée collaboratif, en partie géré par les visiteurs qui peuvent attribuer des mots-clés aux œuvres, les fixer sur une carte, les structurer et les commenter. Cependant, d'autres initiatives semblent correspondre encore bien davantage à l'idée d'une véritable participation des individus.

\section{Le visiteur, nouvel « artiste » créateur d'œuvres ?}

Pour Simon (9), la collaboration du public comprend plusieurs étapes évolutives dont la participation de l'individu à la création artistique en tant que telle constituerait le stade ultime d'une démarche muséale 2.0. Certains musées permettent ainsi aux visiteurs d'apporter euxmêmes des pièces de la collection on line se substituant alors, non seulement au conservateur, 
mais également à l'artiste. Il s'agit dès lors pour les musées d'adopter plus directement le concept de consumer made, défini comme «le résultat de la mise en jeu des compétences d'un ou de certains consommateurs afin de modifier ou d'améliorer l'offre des entreprises et d'arriver ainsi à une création originale » (1).

Si l'intégration des consommateurs se développe de plus en plus dans la démarche de création mise en place par les entreprises, une telle optique peut sembler plutôt incongrue dans l'univers muséal. En effet, la préservation de l'autonomie de l'artiste, tout comme celle du conservateur concernant le choix des œuvres à exposer, constitue un principe fondamental guidant les politiques culturelles des institutions muséales. Mais qu'advient-il de cette logique si le visiteur devient lui-même créateur et fournisseur des contenus muséaux ? Dans cette perspective, confier les rênes de la création aux visiteurs laisse entrevoir une nouvelle logique collaborative entre le public et les musées. L'examen des dispositifs développés récemment par les institutions démontre bien cette implication de plus en plus forte des visiteurs dans la définition de certaines formes d'offres artistiques proposées sur Internet. Les exemples sont nombreux et couvrent des formes de création très variées.

Si certains musées encouragent réellement la création par leur public internaute, ils se contentent cependant, de l'intégrer en marge de leur offre artistique centrale. Ainsi, invitentils le public à créer des objets certes culturels, mais destinés à rester dans un rôle périphérique d'accompagnement des œuvres détenues par le musée. Sur son site Internet, le Tate Modern Museum a mis en place un dispositif appelé « Your Tate Track » invitant des musiciens et des groupes amateurs à soumettre une création musicale inspirée de l'une des œuvres de la collection de l'institution (http://www.tate.org.uk/modern/tatetracks/).

Dans d'autres cas, les visiteurs peuvent être expressément sollicités pour créer une œuvre artistique ex-nihilo afin de répondre à un projet culturel spécifique. Ainsi, le musée Malraux du Havre avait lancé en 2009 un appel à contribution photographique autour de la Normandie, dans le cadre de l'exposition « Voyages Pittoresques ». Un blog avait donc été créé en amont invitant les internautes à déposer leur création et à les soumettre aux votes de la communauté afin d'avoir la chance de voir leurs images exposées au sein du musée. Dans une démarche relativement proche, le Tech Museum de San Jose a proposé dans l'univers virtuel Second Life, en décembre 2007, une reconstitution de ses bâtiments, mais complètement vides. Cette initiative désignée sous 1'appellation «The Tech Virtual», toujours en cours, propose au public de remplir le musée virtuel en exposant ses propres créations (http://www.thetech.org/techvirtual). 
Enfin, certaines initiatives concernent des musées qui n'existent que grâce à la participation des visiteurs, seuls créateurs des œuvres exposées. A titre d'exemple, citons le cas du Museu da Pessoa au Brésil, musée totalement contributif, qui collecte des biographies selon l'idée que chaque individu fait partie de l'Histoire et peut donc immortaliser son histoire spécifique dans un réseau de mémoire sociale, de souvenirs collectifs (http://www.museudapessoa.net/).

Ces actions tendent à faire collaborer le public par une participation «immédiate » à la conception des objets mis en scène par les sites muséaux tandis que les exemples concernant les rôles de médiation et de médiatisation passaient par une forme de réinterprétation $a$ posteriori de l'offre (1). Bien entendu, certaines de ces initiatives, derrière un discours fort de réappropriation, ne cherchent en définitive qu'à alimenter un bouche-à-oreille autour de la structure, la co-création d'offre étant utilisée comme un catalyseur pour une meilleure visibilité et accessibilité symbolique du lieu. Pour d'autres musées, il peut s'agir, à travers cette participation directe du public de bénéficier du potentiel de création de ces derniers et de développer un sentiment fort d'appartenance.

\section{Conclusion}

Avant d'apprécier l'importance de ces pratiques managériales et leurs éventuelles incidences sur la relation visiteur - musée, il convient de garder à l'esprit que bon nombre de ces outils sont encore très récents. Comme le note Divard (5), il est difficile de se prononcer, actuellement, sur leur devenir. Les effets de mode qui accompagnent systématiquement l'émergence de chaque dispositif invitent à être prudent dans l'importance qu'il faut leur accorder.

Par ailleurs, si l'utilisation des outils du Web 2.0 favorise toujours plus fortement une intégration du visiteur au cœur de l'offre patrimoniale, cette orientation n'est pas nouvelle pour les musées. Depuis maintenant de nombreuses années, ces derniers ont entamé une métamorphose conduisant à mettre le public au cœur de leur mission. De nombreux responsables muséaux ont ainsi fait de l'activité de diffusion leur mission prioritaire et n'ont pas hésité à mobiliser, notamment in situ, des outils technologiques afin d'attirer toujours plus de visiteurs. De ce point de vue, la mobilisation des outils du Web 2.0 apparaît plutôt comme un accélérateur de phénomènes déjà existants et permet d'atteindre un certain nombre d'objectifs recherchés par les managers: accroître la notoriété de l'institution muséale, développer l'interactivité et la proximité avec le visiteur, créer du trafic à la fois sur le site 
Internet mais également au sein du musée, bénéficier du potentiel de créativité du visiteur... Plus globalement, ils peuvent être considérés comme des outils favorisant la production de nouvelles formes d'expériences et susceptibles de générer une valeur additionnelle pour l'institution et pour le consommateur.

Cependant, au delà de ces objectifs managériaux, la mise en place de ces outils conduit le visiteur à endosser le rôle de «partenaire » de l'institution. En effet, la mobilisation massive des dispositifs 2.0 de la part des musées conduit à l'émergence d'un consommateur hyperacteur qui va, de façon plus égalitaire, communiquer, coopérer voire débattre avec l'organisation muséale (7). Ce «sacre de l'amateur », ni novice, ni professionnel, de plus en plus sollicité par les organisations, reflèterait pour certains le développement d'une véritable démocratisation des compétences (6). Cependant, au delà du changement de statut du visiteur, ces modifications soulèvent un certain nombre d'interrogations concernant la nature de la relation entre le visiteur et l'institution muséale et finalement la position que cette dernière occupe dans l'espace social. De ce point de vue, ces pratiques collaboratives nous semblent capables de remettre en cause deux caractéristiques qui sont au cœur de l'identité d'un musée. Tout d'abord, elles peuvent conduire à une altération du caractère sacré de l'offre muséale. Si la tendance à la désacralisation du patrimoine n'est pas nouvelle (7), les dispositifs participatifs, en favorisant l'intervention du visiteur au sein même de l'offre culturelle, contribuent à l'exacerber. Le propre de l'artefact culturel étant d'être un bien hors du commun, il paraît inévitable d'aboutir à une banalisation de l'offre muséale en encourageant l'appel aux contributions des visiteurs. La création perdrait ainsi sa dimension exceptionnelle. Au-delà de cette désacralisation, il nous semble que la mobilisation de ces techniques peut également entraîner une certaine remise en cause du discours et plus globalement de l'autorité de l'institution. Historiquement, le musée est l'unique voix autorisée en raison de son expertise scientifique et/ou artistique (3). Or cette voix est désormais diluée, voire effacée, au profit de celles des visiteurs. De telles stratégies d'ouverture, sans un contrôle et un projet clairement défini en amont, peuvent conduire finalement à un brouillage des intentions de l'institution muséale et à une perte d'autorité du discours.

$\mathrm{Au}$ final, le recours à ces techniques 2.0 ne risque-t-il pas de remettre en cause plus profondément la légitimité du musée, définie historiquement comme la «figure institutionnelle du montrer » (4) et occupant traditionnellement une position distanciée dans sa relation avec le visiteur? Si cet ancrage semble s'être déjà altéré depuis plusieurs années, notamment sous l'impact de la marchandisation et de la spectacularisation de l'offre muséale, il nous semble que l'empreinte de plus en plus forte de la logique participative contribue à 
fragiliser encore davantage cette situation. Il convient dès lors de se demander si nous ne sommes pas au début d'une restructuration plus profonde du champ muséal qui imposera de repenser à terme sa propre définition.

\section{Références}

(1) Cova B. (2008), Consumer made : quand le consommateur devient producteur, Décisions Marketing, 50, 19-27.

(2) Cova B. et Cova V. (2009), Les figures du nouveau consommateur : une genèse de la gouvernementalité du consommateur, Recherche et Applications Marketing, 24, 3, 81-100.

(3) Crenn G. (2003), Rhétorique de la transparence et légitimité muséale : à propos de 3 expositions d'ethnologie, Quaderni, 52, 93-103.

(4) Deloche B. (2001), Le musée virtuel, Paris, P.U.F.

(5) Divard R. (2010), Le marketing participatif, Paris, Dunod.

(6) Flichy P. (2010), Le sacre de l'amateur, Sociologie des passions ordinaires à l'ère numérique, Paris, Le Seuil-La République des Idées.

(7) Gombault A. (2009), Le marketing du patrimoine culturel, in Marketing de l'art et de la culture, Bourgeon-Renault D. (ed.), Paris, Dunod, 175-210.

(8) Le Deuff O. (2006), Folksonomies, BBF, 2006, 4, 66-70.

(9) Simon N. (2010), The Participatory Museum, published by Museum 2.0, Santa Cruz, California.

(10) Trant J. (2009), Tagging, Folksonomy and Art Museums: Results of steve.museum's research, University of Toronto / Archives \& Museum Informatics, January.

(11) Vidal G (2006), Contribution à l'étude de l'interactivité, les usages du multimédia de musée, Presses Universitaires de Bordeaux, Pessac. 